\section{Seronegative Spondyloarthritis and Magnetic Resonance Imaging}

To the Editor:

Troppmann and Karsh report a study of 29 patients with spondyloarthritis $(\mathrm{SpA})$ in which they conclude that the requirement for magnetic resonance imaging (MRI) could present an additional barrier to timely treatment with anti-tumor necrosis factor (anti-TNF) agents in 12 (41\% of the cohort evaluated) patients with $\mathrm{SpA}$ in $\mathrm{Canada}^{1}$. There are significant concerns with this conclusion.

Of these 12 patients, 7 had a Bath Ankylosing Spondylitis Disease Activity Index $($ BASDAI) $\geq 4$, with low acute-phase reactants, and 5 had low BASDAI $(\leq 4)$ but elevated acute-phase reactants. The Spondyloarthritis Research Consortium of Canada treatment recommendations ${ }^{2}$ for access to anti-TNF require at least 2 out of 3 of the following: BASDAI $\geq$ 4, elevated acute-phase reactants, and MRI features of inflammation in sacroiliac joints or spine. So it is correct that these 12 patients would not meet the criteria for access to anti-TNF. However, several issues are not addressed in the discussion of these observations.

The most important issue is that it is not clear how many of these patients actually required treatment with anti-TNF therapy. For instance, the 5 patients in the group with a BASDAI $\leq 4$ and elevated acute-phase reactants had a mean BASDAI of only 2.7! How many of these patients would actually be considered candidates for anti-TNF therapy on clinical grounds? Is the cost of such treatment justified, potentially for several decades, in the absence of any additional features of active disease? What other objective measures of disease would be more helpful than an MRI to verify the presence of active disease?

It is also unclear how many of the 7 patients with a BASDAI $\geq 4$ but normal acute-phase reactants would actually be considered candidates for anti-TNF therapy. A previous report has shown that as many as $40 \%$ of patients with a BASDAI $\geq 4$ reported being in a patient-acceptable symptom state (PASS) ${ }^{3}$. Moreover, the BASDAI does not discriminate between mechanical and inflammatory causes of back pain. Consequently, does the absence of any objective measure of active disease constitute justifiable grounds for longterm therapy with anti-TNF agents?
The aim of treatment recommendations is to set standards of care. In each of these 2 circumstances the MRI would have provided useful information to the clinician that would have guided the decision for or against treatment with anti-TNF agents. The conclusion that $41 \%$ of patients may have treatment delayed, inadvertently prolonging disease activity and increasing disability due to lack of access to MRI, seems grossly exaggerated, since in neither circumstance was evidence provided that any of these patients were actually considered for anti-TNF therapy on clinical grounds. MRI is not a requirement for treatment with anti-TNF agents in the province of Ontario and it would be interesting to hear from the authors how many of these patients were actually considered for and then received treatment.

WALTER P. MAKSYMOWYCH, MD, University of Alberta-Medicine, 562 Heritage Medical Research Building, University of Alberta, Edmonton, Alberta T6G 2S2, Canada. Address reprint requests to Prof. Maksymowych; E-mail: walter.maksymowych@ualberta.ca

\section{REFERENCES}

1. Troppmann L, Karsh J. The percentage of patients with seronegative spondyloarthritis requiring magnetic resonance imaging to meet the Canadian Rheumatology Association/Spondyloarthritis Research Consortium of Canada guidelines for access to anti-tumor necrosis factor treatment. J Rheumatol 2008;35:658-61.

2. Maksymowych WP, Gladman D, Rahman P, et al. The Canadian Rheumatology Association/Spondyloarthritis Research Consortium of Canada treatment recommendations for the management of spondyloarthritis: a national multidisciplinary stakeholder project. J Rheumatol 2007;34:2273-84.

3. Maksymowych WP, Richardson R, Mallon C, van der Heijde D, Boonen A. Evaluation and validation of the patient acceptable symptom state (PASS) in patients with ankylosing spondylitis (AS). Arthritis Rheum 2007;57:133-9.

J Rheumatol 2009;36:1; doi:10.3899/jrheum.080721 